IC Vol. $5 N^{\circ}$ 2: pp. 253-266, 2014

\title{
LA EVASIÓN TRIBUTARIA Y SU CONSECUENCIA EN EL DESARROLLO ECONÓMICO DEL PAIS
}

\author{
TAX EVASION AND ITS CONSEQUENCE ON ECONOMIC \\ DEVELOPMENT OF THE COUNTRY
}

Roberto Carlos López Córdoval

\section{RESUMEN}

El presente trabajo de investigación denominado La evasión tributaria y su consecuencia en el desarrollo económico del país, se ha desarrollado usando la metodología de revisión bibliográfica y documental, bajo la perspectiva de que la información recolectada es la que verdaderamente nos informa acerca de la situación relacionada con el tema de investigación. Para ello se ha recurrido a documentos, como tesis y direcciones de Internet, para la recolección de información.

El tema de investigación ha dado como resultado el conocimiento de las múltiples causas de cómo la evasión tributaria es un mal que aqueja no solo a nuestro país, sino también a aquelos que utilizan tecnologías avanzadas para detectar dicho problema.

Se concluye que la evasión tributaria solo se evitará cuando se tome conciencia de que la tributación no es una obligación sino un deber con el Estado. Asimismo, si un estado es considerado como una gran empresa, necesita recursos para llevar a cabo su gestión, y estos recursos se consiguen gracias a la tributación.

PALABRAS CLAVE: Desarrollo económico, evasión tributaria y tributos.

* Recibido: 12 agosto 2014; aprobado: 19 noviembre 2014.

1 Bachiller en Ciencias Contables y Financieras, Asistente contable de la Junta de Usuarios del Subdistrito de Riego Nepeña. E-mail: lopez_cordova_roberto@hotmail.com 


\section{ABSTRACT}

This research used literature review and documentary under the perspective that the information collected truly informs us about the situation related to the subject of research. So, we searched documents like thesis and Internet addresses for collecting information. By this we knew the multiple causes of tax evasión and its widespread consequences in many countries. Its advanced technologies to detect the problema is also a matter of concern.

It is concluded that tax evasion would avoid when all of us aware that taxation is not an obligation, but a duty with the State. Also, if a State is considered to be a large company, you need resources to carry out its management, and these resources are achieved thanks to the taxation.

KEY WORDS: Economic development, tax evasion, and tributes.

\section{INTRODUCCIÓN}

El tema de investigación, denominado "La evasión tributaria y su consecuencia en el desarrollo económico del país", tiene una gran importancia dado que es una problemática que trasciende nuestras fronteras. A continuación detallamos algunas caracterizaciones de la problemática de la evasión tributaria y sus consecuencias en la economía de nuestro país.

En el Perú, la recaudación de los impuestos se ha venido incrementando de manera sostenida en la última década, mostrando un pequeño descenso en el 2009 debido a la menor producción causada por la crisis mundial. Estas mejoras en la economía se reflejan directamente en los impuestos recaudados, tanto de las actividades productivas de las personas como de las empresas, siendo los sectores de minería, construcción y la agroexportación los que contribuyen a tal crecimiento. Por tanto, cuando la economía crece como producto del mayor empleo, producción, exportación o consumo de la población, se generan mayores ingresos y una recaudación más alta de impuestos. No obstante, para llegar a tal situación, el Estado debe buscar la formalización de la economía, ya que gran parte de ella se maneja en el sector informal, razón por la cual no declaran el producto de sus actividades y, por lo tanto, evaden impuestos.

Diario La Primera (2013). La riqueza de una persona o empresa debe ser gravada mediante el Impuesto a la Renta. Pero como en el Perú tenemos una economía distorsionada suceden estas cosas, como que los impuestos indirectos sean el principal mecanismo de recaudación. En su primera presentación ante la Comisión de Economía del Congreso de la República, Tania Quispe Mansilla, jefa de la Superintendencia Nacional de Administración Tributaria (Sunat), reconoció que los grandes problemas a solucionar durante su gestión son com- 
batir los altos niveles de evasión y elusión tributaria en el país, así como recuperar la buena imagen del recaudador mellada en los últimos años. Según cifras del ente recaudador, la evasión en lo referido al Impuesto General a las Ventas (IGV), en la última década pasó de 49,1\% (2001) a 34,7\% (2010). Al analizar el último quinquenio bajo la administración aprista, se aprecia que la evasión fue de $38,8 \%$ (2006) a 34,7\% (2010); es decir, una disminución de 4,1\%. Mientras que en la administración toledista se aprecia una disminución de $6,8 \%$ (de $49,1 \%$ el 2001 a $42,3 \%$ el 2005). Además, el nivel de contrabando alcanzó el $1,8 \%$ de las importaciones totales de bienes (el 2009 representó 2,2\%). De acuerdo a cifras de la Comisión Económica para América Latina y el Caribe (CEPAL) y La Superintendencia Nacional de Aduanas y de Administración Tributaria (SUNAT), el Perú es uno de los países que tiene las tasas más altas de evasión en el IGV en la región. Mientras que en el Perú la evasión alcanzó $34,7 \%$, en Chile y Uruguay representan el 18\%; en Argentina y México, 20\%; Colombia, 22\%; Ecuador, 32\% . Por encima del Perú se encuentra Venezuela, donde la evasión alcanza $66 \%$. Pero no solo se evade el pago del IGV; también se busca la manera de no pagar el Impuesto a la Renta (IR). Sobre este último tributo, el especialista en temas tributarios, Jorge Manini, estimó que aproximadamente la evasión alcanza el $47 \%$ de contribuyentes. Y de acuerdo a un estudio de la CEPAL, la evasión en renta es de $53 \%$ en las personas jurídicas o empresas y de 33\%, en las personas naturales. Es decir, la evasión es más grande en las empresas. El especialista Manini Chung afirma que en una adecuada estructura tributaria, los impuestos directos como el IR son la principal fuente de recaudación de recursos del Estado y los impuestos indirectos (como el IGV o ISC) son la segunda fuente de ingresos, pero esto no sucede en el Perú.

Allendy (2012). La evasión tributaria es toda acción que violando las leyes tributarias, produce la eliminación o disminución de la carga tributaria. La doctrina es general en señalar a la intencionalidad o fraude, según los penalistas, como la condición indispensable para la existencia de este delito, que es una forma de defraudación tributaria. La evasión es plasmada como un delito en nuestro ordenamiento legal, implica que al margen de la norma el contribuyente, es obligado a abonar un porcentaje de sus ingresos al Estado. Opta por no hacerlo, corriendo el riesgo, cada vez más frecuente, de ser detectado por la administración y posteriormente sancionado. Uno de los grandes problemas en nuestro país por la evasión tributaria esta ocasiona menor ingresos fiscales, limitando el cumplimiento de las funciones de Estado por los menores recursos que se dispone. El problema cuando adquiere mayor gravedad, se patentiza en 
el accionario deficiente de los recursos en la incoherencia de los planes económicos, en el poco desarrollo económico y en la desigualdad en la distribución de la renta. De igual manera, también perjudica al Estado la elusión tributaria, que consiste en pagar menos tributos, utilizando las disposiciones legales de forma que signifiquen una menor carga tributaria. En otras palabras, la elusión es el acto no prohibido por la ley, mediante el cual los contribuyentes buscan la manera de pagar menos tributos o no pagarlas.

Latínez, L., (2012). La Evasión Tributaria en el Perú es un “deporte nacional", porque se da en todos los tributos. En este artículo comentaré la evasión tributaria en las distintas categorías del Impuesto a la Renta (IRTA). En el impuesto a la renta existen cinco (5) categorías.

El Impuesto a la Renta (IRTA) es un impuesto directo (progresivo), porque afecta más a las rentas altas que a las bajas. En cambio el Impuesto General a las Ventas (IGV) es un impuesto indirecto (regresivo), porque afecta igual a ricos y a pobres, haciéndolo de modo más fuerte a los pobres, que ahorran poco o nada, que a los ricos, que ahorran más y no pagan el IGV por este ahorro. Así, por ejemplo, un IGV de $18 \%$ afecta en un $18 \%$ a una persona que consume toda su renta, y en un $9 \%$ a una persona más rica, que se gasta en consumo la mitad de su renta y ahorra la otra mitad, por la que no paga el impuesto.

En nuestro país la mayor recaudación tributaria proviene de la recaudación del IGV y en menor proporción, vía recaudación del IRTA, demostrándose de esta manera lo injusto y nada equitativo que es nuestro Sistema Tributario Nacional.

Son rentas de $1^{\text {a }}$ categoría:

*Arriendo y subarriendo de inmuebles (amoblados y sin amoblar), *Arriendo y subarriendo de bienes muebles (maquinaria, autos, computadoras, etc.), *Ceder en uso gratuitamente Inmuebles y bienes muebles.

Las personas naturales perceptoras de rentas de $1^{\mathrm{a}}$ categoría que alquilan y subarriendan bienes muebles e inmuebles tienen que efectuar pagos a cuenta del IRTA y presentar su Declaración Jurada Anual del IRTA y pagar el IRTA anual, previa deducción de los pagos a cuenta del IRTA. Los que ceden gratuitamente bienes muebles e inmuebles tienen que presentar su Declaración Jurada Anual del IRTA y pagar el IRTA anual.

La mayoría de perceptores de rentas de $1^{\text {a }}$ categoría no pagan tributos.

Son rentas de $2^{\text {a }}$ categoría, entre otras, las siguientes:

* Los intereses originados en la colocación de capitales, cualquiera que sea su denominación o forma de pago, tales como los producidos por títulos, cédulas, debentures, bonos, garantías y créditos privilegiados en dinero o en valores. 
* Las regalías. Se considera regalía a toda contraprestación en efectivo o en especie por el uso o por el privilegio de usar patentes, marcas, diseños o modelos, planos, procesos o fórmulas secretas y derechos de autor de trabajos literarios, artísticos o científicos, así como toda contraprestación por la cesión en uso de los programas de instrucciones para computadoras (software) y por la información relativa a la experiencia industrial, comercial o científica. El producto de la cesión definitiva o temporal de derechos de llave, marcas, patentes o similares; las rentas vitalicias, la ganancia de capital.

Los perceptores de rentas de 2 da categoría efectúan pagos definitivos del IRTA por las ganancias de capital por ventas de inmuebles; Retenciones del IRTA definitivos por intereses y regalías; Pagos a cuenta del IRTA y Retenciones a cuenta del IRTA por Ganancia de capital por venta de valores mobiliarios.

Sólo se presenta Declaración Jurada Anual del IRTA por la Ganancia de Capital por la venta de valores mobiliarios.

A las personas naturales que perciben rentas de $2^{\mathrm{a}}$ categoría por intereses y regalías no les retienen el impuesto a la renta.

Las rentas de $3^{\text {a }}$ categoría se refieren a las personas naturales con negocio (bodegas, bazar, panadería, etc.) y a las personas jurídicas.

Los perceptores de rentas de $3^{\text {a }}$ categoría pueden estar en el RUS, RER o en el Régimen General. Los del RUS y los del RER no son fiscalizados y además los controles que se establecieron para monitorearlos, como son el consumo de energía eléctrica y telefónico, han sido dejados de lado, manteniéndose altísima la evasión tributaria.

En el caso de los contribuyentes que están en el Régimen General, existen diversas modalidades de evasión tributaria, que muchas veces lindan con el delito tributario. Así tenemos, venta de mercadería con guías de remisión, compra de facturas, compra de mercadería sin comprobante de pago y su respectiva venta sin comprobante de pago; excesivo gastos por depreciación, manipulación de los costos, manipulación de los libros contables, etc. La SUNAT descuidó la fiscalización del IRTA de $3^{\text {a }}$ debido a la falta de especialistas, pues los mismos fueron separados durante la dictadura de Fujimori.

Recién a partir del 2007 están fiscalizando, pero desconocen la auditoría de este tributo. Se debe precisar que hasta julio de 1990 los auditores de la SUNAT revisaban las remesas de divisas efectuadas por grandes empresas extranjeras, como la Occidental Petroleum Corporation, que remesaba millonarias utilidades en exceso a su principal. También fiscalizaban a las empresas extranjeras, detectándose evasión tributaria, razón por la cual les acotaban 
millonarias resoluciones de determinación y resoluciones de multa. La SUNAT, que se había creado en el año 1989 sobre la base de la Dirección General de Contribuciones y del Instituto de Administración Tributaria (IAT), contaba con los especialistas del caso.

En el caso de los principales contribuyentes, la SUNAT manifiesta que los tiene controlados, pero solo a los que declaran, más no a los que no han declarado.

Son consideradas rentas de $4^{\mathrm{a}}$ categoría el ejercicio individual de cualquier profesión, arte, ciencia, oficio o actividades no incluidas expresamente en la tercera categoría. El desempeño de funciones de director de empresas, consejeros regionales, regidores municipales, síndico, mandatario, gestor de negocios, albaceas y actividades similares.

En el caso de los perceptores de renta de $4^{\mathrm{a}}$ categoría, la evasión tributaria es altísima, muchos por desconocimiento; tal es el caso de los profesionales ajenos a la contabilidad, pues no saben cuándo tienen que hacer los pagos a cuenta del IRTA, y otros, porque no quieren pagar. Un número significativo no entrega comprobante de pago, como es el caso, entre otros, de los abogados, médicos y dentistas.

En cuanto a las rentas de $5^{\text {a }}$ categoría, están comprendidos los que trabajan en forma dependiente; es decir, los que están en planilla. En esta renta también existe una altísima evasión tributaria, que se demuestra con la cantidad de trabajadores que, debiendo estar en planilla, pues son trabajos de carácter permanente, son obligados por los empresarios a entregar recibos de honorarios profesionales. No sólo hay evasión en el impuesto a la renta de $5^{\text {a }}$ categoría, sino también en ESSALUD y en ONP (Sistema Nacional de Pensiones).

Camargo, D., (2005). La evasión tributaria o fiscal es la disminución de un monto de tributo producido dentro de un país por parte de quienes estando obligados a abonarlo no lo hacen; en cambio, sí obtienen beneficios mediante comportamientos fraudulentos. También se puede definir como el incumplimiento total o parcial por parte de los contribuyentes en la declaración y pago de sus obligaciones tributarias. Hay quienes la definen como el acto de no declarar y pagar un impuesto en contradicción con la ley, mediante la reducción ilegal de los gravámenes por medio de maniobras engañosas.

Diario Perú 21, (2013). La Superintendencia Nacional de Aduanas y Administración Tributaria de Perú (SUNAT) informó que en los últimos días ha sancionado a 3152 administradores de gasolineras, hostales y concesionario de universidades de Lima por no entregar comprobantes de pago por las ventas y servicios realizados. El castigo se dio por haber incurrido en una modalidad de 
evasión al no haber declarado la totalidad de sus ingresos y, en algunos casos, se aplicó a un mismo contribuyente por cuarta vez consecutiva, precisó en un comunicado. Las intervenciones a empresas son parte de una estrategia para combatir la evasión del pago de Impuesto General a las Ventas (IGV) y el Impuesto a la Renta (IR) en el sector transporte, donde se ha detectado a contribuyentes que adquieren comprobantes que los grifos dejan de entregar a taxistas y vehículos particulares para incrementar, de manera indebida, sus gastos. Aunque no ofreció montos precisos, la SUNAT informó a inicios que la recaudación tributaria creció un 6,6\% en agosto, gracias al dinamismos de los sectores vinculados a la demanda interna, que permitió compensar los menores ingresos por las exportaciones mineras.

Camargo, D., (2005). La evasión tributaria tiene denuncias y puede ser de dos clases: veraz o infundada. Veraz, cuando obedece a información seria y oportuna del denunciante, e infundada, cuando la denuncia está motivada por el deseo de perjudicar al denunciado más que aportar información valiosa a la administración tributaria. Quien es denunciado se sorprende cuando llegan los funcionarios fiscalizadores a practicar el allanamiento en su domicilio fiscal de manera intempestiva. Si nunca había tenido esa experiencia, es posible que confiese sus faltas, o la presión que sienta lo persuada a reconocerlas, a menos de que crea que por más inspección que le practiquen no van a encontrar evidencias y decida mantener una actitud displicente. El factor sorpresa permite determinar las posibles irregularidades de quienes son evasores, porque la prueba casi siempre está de cuerpo presente y no ha tenido tiempo de ocultarla, a menos que disponga de una estrategia específica.

Diario El Comercio, (2013). La Superintendencia Nacional de Aduanas y de Administración Tributaria (SUNAT) informó hoy que la mala práctica de considerar gastos personales como gastos propios de una empresa, representa un monto de evasión tributaria superior a los S/.2.200 millones. Ante esta situación, el ente recaudador programó más de 2.500 acciones de fiscalización selectiva dirigida a los contribuyentes, donde se ha detectado esta modalidad de evasión, cifra que será incrementada en los siguientes meses. Asimismo, refirió que llevó a cabo una campaña en los medios de comunicación masiva para sensibilizar a la población sobre la importancia de eliminar la mala práctica adoptada por algunos contribuyentes de solicitar factura al momento de adquirir bienes y servicios para su consumo personal o familiar. Estos gastos no son deducibles del Impuesto a la Renta ni le otorgarían al contribuyente derecho a crédito fiscal del Impuesto General a las Ventas (IGV). Resaltó que si un contribuyente ha 
incluido gastos personales en la contabilidad de su empresa, debe rectificar de manera voluntaria sus declaraciones juradas, pagar el importe del impuesto (IGV y Renta) dejado de pagar. Además, por las infracciones cometidas podrá acogerse al Régimen de Gradualidad con una rebaja del $95 \%$, a fin de regularizar su situación antes de ser detectado por la SUNAT.

La evasión tributaria es un común denominador en el mundo, en el Perú y por ende, en nuestra región, siendo un ilícito que es practicado por las personas naturales y jurídicas; por tanto, se enuncia el siguiente problema.

\section{PROBLEMA}

¿Qué consecuencia provoca la evasión tributaria en el desarrollo económico del país?

\section{OBJETIVOS}

Objetivo general: identificar y describir la evasión tributaria y su consecuencia en el desarrollo económico del país.

Objetivos específicos: identificar la evasión tributaria y su consecuencia en el desarrollo económico del país y, describir la evasión tributaria y su consecuencia en el desarrollo económico del país.

A nivel mundial, los impuestos representan la forma de obtener ingresos por parte del estado y a través de sus diferentes órganos, así como también la manera que tienen los ciudadanos para contribuir con el gasto público; sin embargo, no todas las empresas cumplen con la obligación tributaria, ya que hay casos de evasión, lo cual es un tema que preocupa.

Por ende, el presente trabajo de investigación se centró en la evasión tributaria y su consecuencia en el desarrollo económico del país.

En tal sentido, se ha estudiado dicho tema para saber la consecuencia que provoca la evasión tributaria en el desarrollo económico del país.

Por consiguiente, cabe resaltar que hoy en día existen entidades que no cumplen con sus obligaciones tributarias, las cuales causan efecto en la economía social, de manera que disminuyen los ingresos públicos destinados para la elaboración de actividades en bien de la sociedad.

Con el aporte teórico de varios autores, esta investigación ha servido de base para constatar que la tributación es muy importante para atraer recursos económicos al erario público o fisco, como parte de los ingresos públicos, que serán utilizados en obras públicas, traducidos como gastos públicos, hoy en día se han podido lograr grandes obras para beneficio de nuestra comunidad y por tanto, beneficio también para las empresas. 


\section{METODOLOGÍA}

\section{TIPO Y NIVEL DE LA INVESTIGACIÓN DE LA TESIS}

Teniendo en cuenta la complejidad para la recolección de información de carácter económico y financiero en las entidades, debido a que algunas empresas guardan en absoluta reserva la información de sus actividades, el método de investigación aplicado es la revisión bibliográfica y documental.

El tipo de investigación es bibliográfica, debido a que solo se limitó a la investigación de datos de las fuentes de información originales, sin entrar en detalle de cantidades.

El nivel de estudio es descriptivo, debido a que se realizó un estudio de características, cualidades y atributos del tema de investigación sin entrar a los grados de análisis cuantitativos del problema.

\section{DISEÑO DE LA INVESTIGACIÓN}

Según Trochim, un diseño de investigación es: "El diseño de investigación puede ser pensado como la estructura de la investigación, es el pegamento que sostiene juntos a todos los elementos en el proyecto de investigación".

Arias (2004) considera a la investigación documental como el proceso basado en la búsqueda y análisis de datos secundarios; es decir, datos registrados por otros investigadores en fuentes documentales, impresas, audiovisuales o electrónicas.

La investigación realizada buscó la información proveniente de propuestas, comentarios, análisis de temas parecidos al nuestro, las cuales se analizaron al determinar los resultados.

El diseño de investigación aplicado es no experimental debido a que la información recolectada se encontró en las fuentes de información documental.

\section{EL UNIVERSO O POBLACIÓN}

De acuerdo al método de investigación bibliográfica y documental, no es aplicable a alguna población ni muestra.

\section{PLAN DE ANÁLISIS}

De acuerdo a la naturaleza de la investigación, el análisis de los resultados se realizó teniendo en cuenta la comparación a los comentarios o estudios realizados en las informaciones recolectadas. 


\section{TÉCNICAS E INSTRUMENTOS DE RECOLECCIÓN DE INFORMACIÓN}

La técnica que se aplicó en la investigación es la de Revisión Bibliográfica y Documental, mediante la recolección de información en fuentes, como textos, revistas, tesis, artículos periodísticos e información de Internet. Quedó a criterio del investigador la aplicación de instrumentos, como encuestas o entrevistas a fin de obtener de fuente directa; resultados que permitan complementar los bibliográficos y realizar los contrastes con los antecedentes y las bases teóricas planteadas.

A continuación damos el aporte de algunos autores con respecto a la aplicación de esta técnica:

Según Fidias G. Arias (2006, Pág.31: Metodología de la Investigación), "La investigación documental es un proceso basado en la búsqueda, recuperación, análisis, crítica e interpretación de datos secundarios, es decir, los obtenidos y registrados por otros investigadores en fuentes documentales: impresas, audiovisuales o electrónicas".

Amador (1998) afirma que el proceso de revisión bibliográfica y documental comprende las siguientes etapas: consulta documental, contraste de la información y análisis histórico del problema.

En cambio Latorre, A., Rincón, D. y Arnal, J., (2003, pág. 58), a partir de Ekman (1989), definen a la revisión documental como el proceso dinámico, que consiste esencialmente en la recogida, clasificación, recuperación y distribución de la información.

Como salvedad al proceso de recolección de información, Lorenzo, Martínez y Martínez, 2004, advierte que no resulta funcional que un investigador utilice un número excesivo de fuentes de información, sino que debe ser capaz de seleccionar aquellas que mejor respondan a sus necesidades e intereses; es decir, tienen que estar relacionados al tema de investigación.

En cuanto a los instrumentos utilizados, teniendo en cuenta las fuentes de información documentaria y bibliográfica, como textos, tesis, páginas de internet entre otros, se elaboró fichas bibliográficas como instrumento de ubicación de la información.

Al aplicar la Técnica de la Recolección de Información se recurrió a las fuentes de información de origen para la obtención de datos, las cuales permitieron formular resultados y que estos últimos fueron contrastados con los antecedentes y las bases teóricas, a fin de determinar el análisis de resultados y posteriormente, formular las conclusiones como cumplimiento de los objetivos planteados. 


\section{RESULTADOS}

A fin de dar cumplimiento a los objetivos de la investigación sobre las consecuencias que provoca la evasión tributaria en el desarrollo económico del país, se ha llegado a los siguientes resultados:

1. El Estado peruano debe buscar la formalización de la economía, ya que el $70 \%$ de la economía peruana se maneja en el sector informal y solo el $30 \%$ en el sector formal, razón por la cual no declaran el producto de sus actividades, y por lo tanto, evaden impuestos.

2. Los grandes problemas a solucionar son: combatir los altos niveles de evasión y elusión tributaria en el país; además, recuperar la buena imagen del recaudador mellada en los últimos años. La evasión en lo referido al Impuesto General a las Ventas (IGV), en la última década pasó del $49,10 \%$ a $34,70 \%$, habiendo una disminución de $14,40 \%$ de evasión en la última década.

3. Uno de los grandes problemas en nuestro país es la evasión tributaria. Esta ocasiona menores ingresos fiscales, limitando el cumplimiento de las funciones del Estado por los insuficientes recursos que dispone, ya que el $78 \%$ de los tributos recaudados financian nuestro presupuesto.

4. En nuestro país, la mayor recaudación tributaria proviene del IGV e ISC $50 \%$ (43\% y 7\% respectivamente), el IR 46\%, Importaciones o aranceles $3 \%$ y otros tributos $3 \%$. Los mayores ingresos del país vienen de impuestos indirectos como el IGV e ISC, tributos que todas las personas pagamos sin distinción del nivel de sus ingresos, demostrándose de esta manera lo injusto y nada equitativo que es nuestro sistema tributario nacional.

5. Las situaciones que contribuyen a la evasión fiscal son: la falta de conciencia tributaria, contribuciones exageradamente altas, la compleja estructura del sistema tributario, la falta de expedición administrativa de las disposiciones fiscales, bajo riesgo de ser detectado, la resistencia al pago de impuestos, falta de liquidez en las empresas, falta de transparencia en el sistema tributario, deseo de obtener beneficios particulares apropiándose del dinero público, desconocimiento de las normas tributarias, predominio de valores personales conservadores y desinterés por adecuarse a la formalización y legalidad, percepción de estar pagando demasiados impuestos, percepción de no estar recibiendo servicios públicos por los impuestos que se pagan, la desconfianza sobre el acierto con que el estado administra los recursos, la inestabilidad tributaria originada por la modificación constante de las normas. 


\section{ANÁLISIS}

La evasión tributaria existe a pesar de los avances de la ciencia y tecnología para evitarlos. Es necesaria una cultura de concientización en el contribuyente para comprender que su aporte es valioso, porque el $78 \%$ de los tributos recaudados financian al Perú y ello es el soporte de la economía del país. La Superintendencia Nacional de Administración Tributaria, a pesar de aplicar mecanismos para evitar la evasión, no logra controlar completamente dicho problema, generándose una reducción en la recaudación presupuestaria del Estado, el cual repercute en contra del desarrollo del país. Tal afirmación es sostenida por Córdova (2010), quien concluye, entre otros, en que la gran mayoría de los comerciante desconocen hacia donde va todo el dinero que se recauda, lo que generalmente es distribuido según el presupuesto de la república, a las diferentes zonas de nuestro país y que la mayor parte de los comerciantes piensan que la causa más importante para ellos es el elevado porcentaje que tiene los impuestos y que, por lo tanto, los lleva a no cumplir con ellas.

Una de las consecuencias de la evasión tributaria consiste en que el $70 \%$ de la economía peruana se maneja en el sector informal y solo el $30 \%$ en el formal, razón por la cual no declaran el producto de sus actividades, y por lo tanto evaden impuestos. Por eso, cuando la economía crece producto del mayor empleo, producción, exportación, o consumo de la población, se generan mayores ingresos y una recaudación más alta de impuestos.

No obstante, para llegar a tal situación, el Estado debe buscar la formalización de la economía. Tal afirmación corrobora lo concluido por Salvador, M., (2008), quien concluye que la aplicación de una variable independiente logra la puesta en práctica de una estrategia que da como resultado, en este caso, una mayor concientización de la población respecto al pago de sus tributos. Con la educación correspondiente una masa de población o una muestra logra el cambio de sus actos cuando se le explica la importancia trascendental de sus aportaciones, la colaboración de los representantes de las personas jurídicas en el procedimiento de gestión y aplicación de los tributos, representa para la administración tributaria la forma de hacerla tanto posible como eficaz, ya que de otro modo le resultaría difícil desempeñar con satisfacción las funciones que a ella se le han encomendado por ley. Esto es así en razón de que los hechos imponibles desde el punto de vista impositivo ocurren, la mayoría de las veces, en la esfera privada del contribuyente, sin que lleguen al conocimiento de las autoridades fiscales de manera directa, con lo cual, se hace necesario el establecimiento y 
exigencia del cumplimiento de una serie de deberes de cooperación por parte de todos, con el único fin de procurar ese conocimiento indispensable y preciso de los hechos importantes para la imposición.

La economía del estado depende de los ingresos tributarios para que pueda cumplir con sus diversas funciones, como la de brindar servicios a toda la nación, con recursos que buena parte de ellos provienen de los impuestos. Por ello, los gobernantes deben ser rigurosos en la administración de los tributos recaudados, atendiendo las contingencias de la población que ha aportado. Es momento que el gobierno sea drástico en hacer cumplir a los contribuyentes con sus obligaciones tributarias; de lo contrario, el estado estará atado de brazos para cumplir con sus obligaciones.

\section{CONCLUSIONES}

$1^{a}$. La evasión tributaria trae como consecuencia la falta de recursos para garantizar los derechos sociales y económicos de los ciudadanos y dificultades para ofrecerles servicios públicos asistenciales y económicos de calidad.

$2^{a}$. La evasión puede ser parcial o total, y aumenta la carga tributaria a los buenos contribuyentes. Esto se debe a que la creación de más tributos o el aumento de las tasas de los tributos ya existentes afectan a los que ya cumplen con sus obligaciones y no a los evasores.

$3^{\mathrm{a}}$. El alto porcentaje de evasión tributaria en nuestro país nos conlleva a falta de desarrollo económico. Esto se traduce en desempleo y retraso tecnológico, exclusión social y escaso desarrollo de capacidades humanas.

$4^{a}$. Se genera déficit fiscal, lo que origina que para cubrirlo se creen nuevos tributos, aumenten las tasas o se obtengan créditos externos, desconfianza de parte de los inversionistas, fuga de capitales y disminución de la inversión privada.

$5^{\text {a }}$ La evasión fiscal afecta de forma significativa al país, provocando con ello que sólo existan recursos para cubrir las necesidades básicas de la población y que no exista desarrollo en el país.

6 . Los recursos del Estado no son suficientes, por lo que se deja de invertir en educación, tecnología, salud pública, infraestructura, etc., lo cual conduce al país a problemas más serios, como el rezago educativo y el desempleo. Estos, a su vez, traen consigo otros problemas sociales, como pobreza, delincuencia y drogadicción, que lejos de ayudar al país, lo perjudican seriamente e impiden su crecimiento. 
$7^{\mathrm{a}}$. La evasión tributaria atenta directamente contra las finanzas públicas, no ingresa una suma que debía haber entrado o se extrae de esta un beneficio indebido, el cual configura el daño inmediato, directo y cuantificable. Visto en forma individual, se trata de un daño patrimonial a la par de uno mediato que, a diferencia del anterior, es inmaterial y constituye un peligro abstracto para el orden socioeconómico, producido por atentar contra la aplicación del principio de justicia fiscal en el reparto de los tributos, como es el deber de solidaridad expresado en la obligación de colaborar con las cargas públicas.

\section{AGRADECIMIENTO}

A la Junta de Usuarios del Sub Distrito de Riego Nepeña, por brindarme las facilidades de poder realizar esta etapa de mi formación profesional.

\section{REFERENCIAS BIBLIOGRÁFICAS}

AllENDY, (2012). Evasión tributaria. Recuperado 06, 2012, de http://www.buenastareas.com/ ensayos/Evasion-Tributaria/4452686.html

CAMARGO, D., (2005). Evasión fiscal: un problema a resolver. Edición electrónica a texto completo en www.eumed.net/libros/2005/dfch-eva/

CAMARgo, D., (2005). Evasión Tributaria tiene denuncias y pueden ser dos clases: Veraz o infundada. Edición electrónica a texto completo en www.eumed.net/libros/2005/dfch-eva/

DiARIO LA PRIMERA. (23 de setiembre de 2013). La evasión tributaria en la mira de la Sunat. http://www.diariolaprimeraperu.com/online/economia/la-evasion-tributaria-en-la-mira-desunat_95332.html.

DIARIO PERÚ 21. (11 de setiembre de 2013). Sunat sanciona a más de 3,000 locales en Lima por evasión. Peru21.pe/economía, pag.5.

DiARIO El COMERCIO. (2013). Solicitud indebida de facturas genera evasión por S/.2.200 millones,[OnLine], Disponible en: http://www.ifac.org/sites/default/ files/downloads/AuditorAsa Financiera_de_PYMES.pdf.

LAtínEZ, L. (2012). Evasión tributaria en el impuesto a la renta. Disponible desde: http://www.voltairenet.org/article176361.htm 